\title{
Necrobiosis lipoidica diabeticorum
}

National Diabetes Information Clearinghouse (NDIC)

\section{Source}

National Diabetes Information Clearinghouse (U.S.). (2009). The diabetes dictionary. [Bethesda, Md.]: U.S. Dept. of Health and Human Services, National Institutes of Health, National Institute of Diabetes and Digestive and Kidney Diseases, National Diabetes Information Clearinghouse.

A skin condition usually on the lower part of the legs. Lesions can be small or extend over a large area. They are usually raised, yellow, and waxy in appearance and often have a purple border. 